\section{Einige explorative Überlegungen zu einer ökologischen Technologiepolitik}

„Die Rede sei vom Nordwest-Lotto in Köln. Es vermarktet ein weithin bekanntes Produkt. Nun hat man sich überlegt, wo eine innovatorische Strategie ansetzen müßte und kam zu dem Schluß, den Umsatz dadurch steigern zu können, daß öfter »Sechs Richtige" getippt werden. Zuerst wurde eine Befragung in Auftrag gegeben. Sie führte zu dem Ergebnis, daß die Gewinner der letzten Jahre sämtlich ihre Tippzettel richtig ausgefüllt und ordnungsgemäß eingereicht hatten. Nur 0,1 vH der Tipper insgesamt hatten die Tippzettel zwar richtig ausgefüllt, aber leider nicht abgegeben. Es lohnte sich also nicht, über die Ausschöpfung dieses kleinen Rests den Umsatz steigern zu wollen. Ein weiteres Ergebnis war, daß die. Zettel mit den Sechsertipps zu $80 \mathrm{vH}$ mit schwarzen Kugelschreibern geschrieben wurden. So kam man auf die Idee, den Tippzetteln schwarze Kugelschreiber beizugeben, um so mit den Erfolgschancen auch den Umsatz zu steigern. Die wirklich zukunftsweisende Idee wird noch kommen: Man wird Roland Berger und Partner beauftragen, Vorschläge für einen ÖkoTipp zu erarbeiten.

Das Beispiel ironisiert die Vergeblichkeit des Bemühens, durch Befragung und deren Auswertung etwas übex erfolgreiches innovatorisches Handeln erfahren zu können. Lehrt doch nur der Wettbewerb selbst die Innovatoren, was sie mit Aussicht auf Erfolg tun können. Aus dem Markt beziehen sie ihr konkretes Erfahrungswissen. Befragungen greifen ins Leere" (Helmstädter 1993, 286).

Dieses Zitat ist aus zwei Gründen interessant: Zum einen bezeichnet es pointiert eine Position, die in der Innovationsdiskussion immer wieder kehrt: nämlich die Annahme der Überlegenheit des Marktes als Entdeckungsverfahrens und die Vergeblichkeit staatlicher Bemühungen, etwa durch Befragungen unentdeckte Innovationspotentiale zu heben und dann zu fördern (vgl. in abgeschwächter Form Erdmann 1993, 215 ff.). Und zweitens ist das Beispiel, wie wir spätestens seit der magischen Wirkung des Jackpots wissen, offensichtlich falsch. Selbstverständlich verfügen die Lottogesellschaften über Strategien, mit denen sie der Kundschaft erfolgreich suggerieren können, daß ihre Gewinnchancen durch Systemspiele respektive die Auszahlung durch den Jackpot größer ausfallen. Wir wissen natürlich nicht, ob diese Strategien Resultat einer Befragung sind, aber sie sind sicher auch nicht das Ergebnis eines reinen Trial and Error Prozesses, an dessen Ende die Selektion des Erfolges durch den Markt steht.

\section{Interventionsmuster der Technologiepolitik}

Idealtypisch lassen sich zwei theoretische $\mathrm{Zu}$ gänge zu einer aktiven Technologiepolitik formulieren. Die neoklassische Vorstellung beruht darauf, daß sich bei Vorliegen von externen Effekten, Transaktionskosten, positiven Skalenerträgen, sunk costs und Spill-over-Effekten zwischen den Einzelmärkten suboptimale Gleichgewichte einstellen. In allen diesen Fällen - sie werden üblicherweise als Ausnahme von der Regel angesehen - liegt Marktversagen vor (Romer 1986, Lucas 1988). Das gilt für die statische wie für die dynamische Betrachtung.

Im Prinzip laufen Argumente dieses Typs darauf hinaus, daß der Innovator nicht in vollem Umfang in den Genuß seiner Innovationsrente kommt oder die Rückflüsse seiner Innovationen mit einem zu großen Risiko versehen sind. In solchen Fällen ist denkbar, daß Innovationen, die im Falle eines perfekten Marktes getätigt worden wären, unterlassen werden. Grundsätzlich könnte es dann die Aufgabe des Staates sein, mit politischen Mitteln dieses Marktversagen zu kompensieren. Das kann die Verbesserung des Patentschutzes ebenso wie die direkte Subventionierung von Technologien sein. Auch die politischen Akteure stehen allerdings ten sich eigennützig. Das kann aus neoklassischer Perspektive zu einer politischen Lösung des Marktversagens führen, die ebenfalls zu einem suboptimalen Gleichgewicht führt. Deshalb läßt sich aus dieser theoretischen Sicht sowohl eine interventionistische Position, die darauf beruht, daß der Politik eine brauchbare Lösung der Informationsprobleme zugetraut wird, wie auch eine antiinterventionistische Position, die auf der gegenteiligen Annahme beruht, begründen.

Die Vorstellung der evolutorischen Ökonomik (vgl. zur allgemeinen Übersicht über den Entwicklungsstand der evolutorischen Ökonomik Witt 1991; und zur Innovationsproblematik Dosi/Freeman/Nelson/Silverberg/Soete 1988) geht demgegenüber davon aus, daß die technologische Entwicklung eine Eigendynamik besitzt, die vergleichsweise unabhängig von den Marktkräften ist. Gefaßt wird diese Eigendynamik mit dem Begriff des »technologischen $\mathrm{Pa}$ radigmas «, an dem sich Forschungseinrichtungen, Unternehmen und Branchen orientieren. Darunter kann man »das grundlegende, generelle Wissen, das als Basis für das Verstehen und Erklären von bestimmten (technischen) Sachverhalten und Abläufen, dient, « (Hanusch/Canter 1993, 20) verstehen. Entscheidend ist, daß solche Paradigmen durch ökonomische, soziale und kulturelle Routinen einen bestimmten technologischen Entwicklungspfad festlegen - in der jüngeren Systemtheorie eine Trajektorie festlegen, an dem sich alle beteiligten Akteure orientieren.

Eine wesentliche Differenz zur neoklassischen Sichtweise weist die evolutorische Ökonomik in vor großen Informationsproblemen und verhal- der Bewertung von Externalitäten auf. Aus neoklassischer Perspektive beeinflußt die Existenz von Externalitäten die Innovationsrente negativ. Um es vereinfacht auszudrücken: Schnelle Imitation durch konkurrierende Unternehmen sorgt dafür, daß die Gewinnmarge des Innovators frühzeitig auf den Branchendurchschnitt eingeebnet wird; dadurch wird der Anreiz, innovativ tätig zu sein, geringer. Demgegenüber postuliert die evolutionäre Ökonomik die Existenz von positiven Synergieeffekten bei der Innovationstätigkeit zwischen Unternehmen, die nicht über Marktbeziehungen ausgetauscht werden (vgl. Nelson/Winter 1982). Empirisch kann man diesen Sachverhalt etwa durch strategische Allianzen zwischen großen Unternehmen bei gemeinsamen F. u. E. - Vorhaben oder durch die freiwillige Schaffung von technologischen Standards, an denen sich eine Vielzahl von Unternehmen ausrichtet, illustrieren. Das ökonomische Risiko des Innovationserfolges wird so auf mehrere Unternehmen umgelegt und die Transaktionskosten können durch Industriestandards gesenkt werden. Allgemein: »Externe Effekte mögen zwar einen negativen Einfluß auf das Anreizsystem der einzelnen Unternehmung ausüben, das aber wird sicherlich durch die zusätzlichen positiven Einflüsse (über-)kompensiert, die sich aus gemeinsam vorangebrachten technologischen Neuerungen ergeben « (Hanusch, Canter 1993, 35).

Diese idealtypische Überzeichnung zweier Positionen führt zu zwei differenten Interventionsmustern der Technologiepolitik. Im einen (neoklassischen) Fall geht es prinzipiell um die Beseitigung von externen Effekten mit dem Ziel, das marktwirtschaftliche Anreizsystem für Innovationen zu fördern. Im anderen (evolutionärökonomischen) Fall geht es um die Förderung von positiven Externalitäten im vormarktlichen Bereich. Das muß nicht unbedingt ein Gegensatz sein; man kann in vielen Fällen das eine im marktlichen Bereich tun ohne das andere im vormarktlichen Bereich zu lassen. Aber unter der Annahme einer harten Budgetrestrikition der öffentlichen Hände wird daraus schnell eine politische Konkurrenz um knappe Haushaltsmittel. Immerhin kann der zweite Ansatz für sich verbuchen, daß die Wissensanforderungen an die politische Intervention etwas geringer sind. Der Staat muß nicht unbedingt wissen, welche konkreten Innovationen durch die Existenz von unerwünschten Externalitäten sich auf dem Markt nicht durchsetzen können und auf die eine oder andere Weise gefördert werden sollten; schwächere Einschätzungen der Art, welche technologischen Paradigmen zukunftsträchtig sind und die Förderung entsprechender F.u.E-Netzwerke reichen aus. Aber auch das ist ein anspruchsvolles Programm.

\section{Schlïsseltechnologien und strategische Sektoren}

Die Annahme, daß positive externe Effekte bei den F.u.E.-Bemühungen im vormarktlichen 
IÖW-Jahrestagung am 25. und 26. November in Hamburg Ökologie und Innovation - Ziele, Blockaden, Bedingungen

Freitag, 25. November

16.00 Uhr Begrüßung der Teilnehmer und Teilnehmerinnen durch Dr. Stefan Zundel, IÖW

Grußwort des Präses der Wirtschaftsbehörde Hamburg - Prof. Erhard Rittershaus

16.30 Uhr »Surfen auf der Modernisierungswelle«, Einführung und Hauptfragestellung Prof. Dr. Arnim von Gleich (FH Hamburg)

17. 30 Uhr Umweltschutz und Wettbewerbsfähigkeit - unvereinbar?! - Dr. Claas van der Linde (Hochschule St. Gallen)

\section{$\mathrm{Zu}$ den einzelnen Themen}

Voraussetzungen und mögliche Reichweite einer Ökologisierung auf dem »Trittbrett« der wirtschaftsstrukturellen Modernisierung. Innovationsbündnisse, Modernisierungsgewinner und -verlierer. (A. von Gleich, FH Hamburg, Lehrgebiet Technikbewertung/ IÖW)

Ökologische Innovation als Strategie zur langfristigen Sicherung des Standorts Deutschland und der internationalen Wettbewerbsfähigkeit der bundesrepublikanischen Wirtschaft. (C. van der Linde, St. Gallen. Van der Linde hat die deutschsprachigen Länder in der von M. E. Porter (Harvard) geleiteten empirischen Studie zu den Voraussetzungen internationaler Wettbewerbsfähigkeit bearbeitet.)

> Voraussetzungen für Innovationsfähigkeit, Beziehungen und Akteure in nationalen und regionalen Innovationssystemen sowie Umrisse einer innovationsfördernden Infrastrukturpoli-
18. $30 \mathrm{Uhr}$ Innovationsfähigkeit und Umrisse einer innovationsfördernden Infrastrukturpolitik - Prof. Dr. Ch. Freemann (University of Sussex, Brighton)

\section{Samstag, 26. November}

9. $30 \mathrm{Uhr}$ Wirtschaftswachstum und Ökologie aus evolutionsökonomischer Sicht Prof. Dr. Ulrich Witt (Universität Freiburg)

10.30 Uhr Natur als Vorbild für ökologische Technik?! - Prof. Dr. Bernd Heydemann (Universität Kiel, angefragt)

tik. (Ch. Freeman, Science Policy Resarch Unit University of Sussex. Freeman ist einer der Begründer der internationalen empirischen Innovationsforschung)

Auf welche theoretischen Konzepte in der Ökonomie kann sich eine > Theorie der Innovation< stützen? Gibt es eine unüberbrückbare Kluft zwischen Neoklassik einerseits und der Schumpeterschen Tradition sowie der >evolutionären Ökonomie< andererseits? (U. Witt, Universität Freiburg)

Können wir von der Natur lernen? Natur als Vorbild für ökologische Technik? (B. Heydemann, Universität Kiel. Als Umweltminister von Schleswig-Holstein Initiator der dortigen $>$ Forschungsstelle für Ökosystemforschung und Ökotechnik «)

Beispiele und Felder konkurrierender Innovationsstrategien:

a) Vom nachsorgenden zum integrierten Um-
11.45 Uhr Vom nachsorgenden zum integrierten Umweltschutz - hemmende und fördernde Bedingungen - Karin Robinet (IÖW)

15. $00 \mathrm{Uhr}$ Innovationen und ökologisches Stoffstrommanagement in der chemischen Industrie - Dr. Stefan Zundel (IÖW)

16.00 Uhr Raumnutzung in der Logistik von der informationstechnischen zur sozialen Innovation - Markus Hesse (IÖW)

17. 15 Uhr Wider die Technologiefixiertheit in der Innovationsdebatte Prof. Dr. Helmar Krupp

weltschutz, fördernde und hemmende Bedingungen (K. Robinet, IÖW )

b) Ökologische Innovationsperspektiven der chemischen Industrie. (S. Zundel, IÖW)

c) Logistik- und Raumnutzungskonzepte am Übergang von der Massen- zur differenzierten Qualitätsproduktion. (M. Hesse, IÖW)

- Innovation und Kommunikation. Wie realistisch sind die Perspektiven einer Integration des ökologischen Diskurses in die Innovationssysteme? Laufen technologieorientierte staatliche Förderprogramme und Unternehmensstrategien zunehmend ins Leere - angesichts eines beobachtbaren Umschlags von der technologiegetriebenen zur nachfrage- bzw. problemgetriebenen Innovation? (H. Krupp, Universität Karlsruhe. Ehemals Leiter des Fraunhofer Instituts für Systemtechnik und Innovationsforschung)

kroelektronik, wird überschätzt. Insbesondere dürfte das Argument einer drohenden strategischen Abhängigkeit von japanischen Herstellern so nicht haltbar sein.

- Netzwerke und positive Netzwerkexternalitäten spielen bei der Invention und Innovation eine große Rolle.

- Die Umwelttechnik spielt bei der empirisch orientierten Identifikation von strategischen Technologien bislang keine nennenswerte Rolle.

\section{Zu einer Technoligiepolitik} mit umweltpolitischen Absichten

Die Rubrizierung der Umwelttechnik unter ferner liefen in den empirischen Studien zu den deutschen technologiepolitischen Erfolgspotentialen steht in einem augenfälligen Gegensatz zu den Wachstumsraten des Umwelttechnik- 
marktes. Das läßt verschiedene Interpretationen zu. Es kann sein, daß die Zuordnung der Identifikationsmerkmale für eine Schlüsselindustrie im Falle der Umwelttechnik an den Unzulänglichkeiten der statistischen Erfassung scheitert. Es kann sein, daß die Umwelttechnik, gemessen am F. u. E. Einsatz, faktisch kein strategisch bedeutsamer Industriezweig ist. Es kann aber auch sein, daß die überwiegend nachsorgend strukturierten Umwelttechniken an bewährte Technologien nur anknüpfen, während das innovative Potential integrierter Umwelttechniken noch überhaupt nicht ausgeschöpft ist. Für alle drei Annahmen lassen sich Belege beibringen, wirklich erhärten läßt sich bislang keine. Und so muß man nüchtern feststellen, daß zwar mittlerweile eine ganze Reihe von wirtschaftswissenschaftlichen Studien über den Umwelttechnikmarkt vorliegen, wir aber über das strategische Erfolgspotential der Umwelttechnik kaum etwas wissen.

Die Umwelttechnik repräsentiert sicherlich auch kein einheitliches technologisches Paradigma. Vielmehr haben wir es mit einer Vielzahl von branchen- und firmenbezogenen technischen Ansätzén zu tun, die je nach Problemstellung hochgradig ausdifferenziert sind. Insofern ist die Idee, daß von der Umwelttechnik Netzwerkexternalitäten ausgehen würden, in dieser Form nicht richtig. D. h. nicht, daß es im Bereich der Umwelttechnik keine Netzwerke geben würde. Im Gegenteil muß man annehmen, daß Innovationen in diesem Bereich regelmäßig in Form einer Kombination von unternehmensspezifischen Prozeß- und ProduktKnow-how mit branchenübergreifenden oder sogar branchenfremden technologischen Entwicklungen vorgenommen werden. Anwendungsspezifische Entwicklungen der Meß-und Regeltechnik sind dafür Beispiele. Es düfte also durchaus Netzwerkexternalitäten geben.

Man darf aber vermuten, daß solche Netzwerke weniger durch die Entwicklung einer bestimmten Umwelttechnologie dominiert werden, als vielmehr durch die Neukombination von technischen Entwicklungslinien in Anwendungskontexten organisiert sind. Weil die Umwelttechnik als Ganze nicht sinnvoll den Begriff der Basistechnologie ausfüllt, muß auch das Problem der positiven Externalitäten hier neu und vermutlich anders als für andere Technologielinien diskutiert werden.

Hier gibt es eine interessante Verbindung zu den jüngsten Bemühungen um ein branchenübergreifendes oder akteurskettenübergreifendes ökologisches Stoffstrommanagement (vgl. Enquete 1994, 609 ff.; zu den Verbindungslinien, die zwischen Innovation und ökologischer Optimierung gezogen werden können auch den Sammelband Hellenbrand/Rubik 1994). Man darf annehmen, daß die positiven Externalitäten, die die evolutorische Ökonomik für das Zusammenwirken mehrerer Akteure bei F.u.E.Bemühungen annimmt, auch für das kooperative Zusammenwirken von Akteursketten bei den Bemühungen um ein Stoffstrommanagement wirksam sind. Hier sind die positiven Netzwerkeffekte gemeinsamer Innovationsbemühungen allerdings nur ein Aspekt unter mehreren und das politische Interventionsmuster muß sicher anders ausfallen und begründet werden als dies der Fall wäre, wenn es lediglich um die Förderung von Innovationen ginge.

Für eine Technologiepolitik mit ökologischen Absichten stellt sich darüberhinaus das Problem, daß der Staat womöglich die Entdeckung von ökologischen Marktnischen subventioniert, wo keine zu finden sind. Immerhin ist der Umwelttechnikmarkt hochgradig von ordnungsrechtlichen Vorgaben bzw. staatlicher Nachfrage abhängig. Das legt die Vermutung nahe, daß entweder staatliche Fördermaßnahmen zu einem vollzugsunterstützenden Mittel der Ordnungspolitik oder aber zu einer Subventionierung des Exports der Umwelttechnik in solche Länder, deren Standards an bundesrepublikanische Maßstäbe angeglichen werden, degenerieren. Die Eindrücke, die man in dieser Hinsicht aus der Lektüre staatlicher Förderprogramme gewinnen kann, sind noch nicht sehr ermutigend (Robinet, Zundel 1994).

Die größte Schwierigkeit dürfte aber darin liegen, daß die Verknüpfung der Technologiepolitik mit umweltpolitischen Absichten nicht nur eine ökonomische Zielsetzung (etwa die Standortsicherung), sondern auch eine ökologische Lenkungsabsicht verfolgt. Sie ist vor das Problem gestellt, die gegenüber den herkömmlichen Lösungen ökologischeren Technologien identifizieren zu müssen. Gemeinhin wird dieses Problem in den Förderprogrammen durch die Einführung des Begriffs der integrierten Umwelttechnik gelöst. Dieser wird dadurch definiert, daß es sich um solche Technologien handele, die bereits die Entstehung von Emissionen, die dann durch end-of-the-pipe-Technologien herausgefiltert werden müssen, verhindern. In der Förderpraxis ist es aber recht schwierig, im Einzelfall festzustellen, ob eine Technologie integriert ist oder nicht. Hier wie generell dürfte wohl gelten, daß die Richtungssicherheit von ökologischen Optimierungsbemühungen in Unternehmen keineswegs gegeben ist. Auch hier existiert noch erheblicher Forschungsbedarf.

En passant ist staatliche Förderpolitik bei diesem Bemühen dann auch wieder mit dem Problem konfrontiert, für ganz konkrete Fördervorhaben ihre Marktgängigkeit evaluieren zu müssen. So wird die Idee der evolutorischen Ökonomik, durch die Förderung weitaus weniger spezifizierter Netzwerkaktivitäten auch die Informations- und Begründungslast staatlicher Förderpolitik zu erleichtern, wieder unterlaufen. Womöglich wäre hier die bereits angedeutete Strategie, die den Innovationsgedanken mit dem ökologischen Stoffstrommanagement verknüpft, erfolgreicher. Dafür spricht auch, daß das technische und soziale Innovationsgebahren existenter Netzwerke in diesem Bereich auch ohne staatliches Zutun vielversprechend ist (vgl. Jänicke 1993)
Schließlich muß auch gefragt werden, ob die Umweltpolitik gut beraten ist, wenn die Förderung von (innovativen) Umwelttechnologien in den großen Rahmen der Standortsicherung und des internationalen Technologiewettbewerbs gestellt wird. Einmal mehr muß dann die Umweltpolitik nachweisen, daß sie auch ökonomisch vorteilhaft ist. Ob sich das »Surfen auf der Modernisierungswelle« (v. Gleich) umweltpolitisch auszahlt, ist einstweilen aber noch eine offene Frage.

\section{Umweltpolitik in dynamischen wirtschaftlichen Systemen}

Die von ökologischem Gedankengut inspirierte Literatur, die sich mit der destruktiven Seite des technischen Fortschritts auseinandersetzt, füllt mittlerweile Bibliotheken. Offensichtlich steht eine Orientierung, die einer Beschleunigung des technischen Fortschritts mit umweltpolitischen Argumenten das Wort redet, der traditionellen Auffassung, wonach technischer Fortschritt erst dann zur Anwendung kommen darf, wenn alle damit verbunden Risiken bekannt und kalkulierbar sind, entgegen. Dabei ist der Gedanke, mit Innovationen einen umweltpolitischen Geländegewinn zu erzièlen, auch bei umweltpolitisch engagierten Autoren keineswegs neu (vgl. Jänicke 1986, 149 ff.). Gleichwohl gilt, daß die teils implizite, teils explizite politische Vorgabe, man müsse den technischen Fortschritt verzögern und anhalten, prima facie nicht zu einer innovativen Orientierung paßt.

Wenn man nicht der wohl unrealistischen Idee anhängt, daß die moderne Industriegesellschaft mit 5 oder 6 Milliarden Menschen wieder auf ein stationäres und stabiles Gleichgewicht mit der Natur heruntergefahren werden kann, bei dem die Technosphäre lediglich ein vernachlässigbar kleiner Bestandteil der Biosphäre ist, dann stellt sich die Frage anders: Es geht dann nicht um das »Ob« des technischen Fortschritts sondern um die Art des technischen Fortschritts. Im Hinblick auf eine Technikentwicklung lassen die bisher vorliegenden Leitbilder ökologischen Wirtschaftens, insbesondere die zahlreichen Versuche, das Konzept sustainable development zu operationalisieren, noch viele Fragen offen. Einige seien der Kürze halber hier angedeutet:

- Neue Technologien beeinflussen die Interdependenz zwischen dem ökologischen und dem ökonomischen System. Negativ, indem sie neue, bislang noch unbekannte ökologische Risiken heraufbeschwören können; u.U. auch positiv, indem sie neue Gleichgewichtsbedingungen zwischen Gesellschaft und Natur schaffen.

- Gegenüber unbekannten Risiken sind die meisten Kriterien und Leitbilder nachhaltigen Wirtschaftens blind (eine interessante Ausnahme ist etwa v. Gleich 1994). Hier geht es u.a. um das Problem, ob technologische Entwicklungen in einem ökologischen und in einem sozialen Sinne reversibel sind, wenn sich im Zuge 
des Erkenntnisfortschrittes herausstellt, daß sie nicht mehr akzebtable Schäden und Risiken heraufbeschwören. Ökologisch reversibel sind sie, wenn es technisch möglich wäre, den status quo ante herzustellen. Sozial reversibel sind sie, wenn die technische Möglichkeit auch mit einem ökonomisch und sozial vertretbaren Aufwand realisierbar wäre. Die Abhängigkeit mancher osteuropäischer Staaten von Atomkraftwerken, deren Sicherheitstechnik völlig ungenügend ist, ist zumindest ein abschreckendes Beispiel für fehlende ökonomische und soziale Reversibilität, vielleicht auch für fehlende technische Reversibität, wenn man an die Entsorgungsprobleme des Atommülls in Betracht zieht.

Ferner sind die Kriterien nachhaltigen Wirtschaftens fast ausnahmslos als Handlungsbeschränkungen formuliert. Die Idee, daß es vielleicht Technologien geben könnte, die die Produktivität der Natur für die Gesellschaft steigern können, ohne sie zu schädigen, ist scheinbar so nachhaltig desavouiert, daß sie in der gegenwärtigen Diskussion um das Konzept des sustainable development keine nennenswerte Rolle mehr spielt. Ob das angesichts des Innovationsschubs bei jenen Biotechnologien, die gleichsam der Natur abgeschaut sind, so bleiben muß, ist zumindest auch eine offene Frage.

Stefan Zundel, Berlin
Literatur

- Dosi, G., Freeman, R. u. a. (Hg.) 1988: Technica Change and Economic Theory. London.

- Enquete-Kommission »Schutz des Menschen und der Umwelt« des Deutschen Bundestages (Hg.); 1994. Die Industriegesellschaft gestalten Perspektiven für einen nachhaltigen Umgang mit Stoff- und Materialströmen. Bonn.

- Erdmann, G. 1993: Elemente einer evolutorischen Innovationstheorie. Tübingen.

- Gleich, A. v. 1994: Sanfte Chemie - Eine Innovationsperspektive für die chemische Industrie. Universitas, 729-741, 49. Jg.

- Gleich, A. v., Lucas, R. 1994: Veränderte Standortanforderungen in einer zukünftigen Unternehmenslandschaft. IÖW-VÖW Informationsdienst $2 / 94$

- Grupp, H., Schmoch, U. 1992: Wissenschaftsbindung der Technik - Panorama der internationalen Entwicklung und sektorales Tableau für Deutschland. Heidelberg

- Hanusch, H., Cantner, U. 1993: Neuere Ansätze in der Innovationstheorie und der Theorie des techischen Wandels - Konsequenzen für eine Industrie und Technologiepolitik. In: Meyer-Krahmer, F. (Hg.): Innovationstheorie und Technologiepolitik, Heidelberg.

- Hellenbrandt, S., Rubik, F. (Hg.) 1994: Produkt und Umwelt. Anforderungen, Instrumente und Ziele einer ökologischen Produktpolitik. Marburg.
- Helmstädter, E. 1993: Berichterstattung zum Abschnitt II: Erfolgsfaktoren im Innovationsprozeß. In: Meyer-Krahmer, F. (Hg.): s.o.

- Jänicke, M. 1986: Staatsversagen. Die Ohnmach der Politik in der Industriegesellschaft. München

- Jänicke, M. 1993: Über ökologische und politische Modernisierungen. Zeitschrift für Umweltpolitik und Umweltrecht, 159-175

- Legler, H., Grupp, H., Gehrke, B., Schasse, U. 1992: Innovationspotential und Hochtechnologie - Technologische Position Deutschlands im internationalen Wettbewerb. Heidelberg

- Lucas, R. 1988: On the Mechanics of Economic Development. Journal of Monetary Economics, 3-44, Vol 22 .

- Meyer-Krahmer, F. 1992: Strategische Industrien im internationalen Vergleich: Arbeitsteilung und politische Instrumente. In: Fricke, W. (Hg.): Jahrbuch Arbeit und Technik 116-126

- Nelson, R., Winter, S. G. 1982: An Evolutionary Theory of Economic Change. Boston, Mass.

- Robinet, K., Zundel, S. 1994: Förderinstrumente und wirtschaftliche Entwicklung. Gutachten im Auftrag des Büros für Technikfolgenabschätzung (unveröffentlicht). Berlin.

- Romer, P. M. 1986: Increasing Returns and Long-run Growth. Journal of Political Economy, 1002-1037, Vol. 94.

- Witt, U. 1991: Reflections on the Present State of Evolutionary Economic Theory. In: Hodgson, G./Screpanti, E. (Hg.): Rethinking Economics: Markets, Technology, and Economic Evolution, 83-102

\section{Effizienz und Risiko}

\section{Vorausschauende Technikbewertung am Beispiel: Umwelteinflüsse Neuer Werkstoffe}

Die Debatte um >sustainability< hat noch einmal die Notwendigkeit einer gewaltigen Reduzierung der Stoff- und Energieströme vor allem in den Industriestaaten deutlich gemacht. Nicht nur Verhaltensänderungen (Sparsamkeit) und Produktoptimierungen (Langlebigkeit), sondern auch technische Innovationen mit dem Ziel einer > Effizienzrevolution< (v. Weizsäcker) sollen zu diesem Ziel beitragen. Gerade bei diesen technischen Innovationen besteht allerdings die Gefahr, daß die höhere Effizienz durch Erhöhung der Eingriffstiefe in Naturzusammenhänge erzielt wird. Auf diesem Wege könnten geradezu proportional mit der Material- und Energieeffizienz auch die technischen und ökologischen Risiken steigen.

Die Kernenergie wird schon mit Blick auf die $\mathrm{CO}_{2}$-Problematik neu diskutiert. Viele gentechnische Verfahren sind tatsächlich um Zehnerpotenzen material- und energieffizienter als chemisch-synthetische oder klassisch biotechnologische, und auch die ökologische Wünschbarkeit des Einsatzes von Kunststoffen im Karosseriebau bzw. von Hochleistungskeramiken im
Motorenbau wird vor allem mit einer erwartbaren Verminderung des Treibstoffverbrauchs begründet:

Es mehren sich die Anzeichen dafür, daß wir in vielen technischen Bereichen vor neuen Konflikten über den einzuschlagenden Entwicklungspfad, über die konkrete stofflich-technische Ausprägung der notwendigen >ökologischen Modernisierung ‘ stehen. Mögliche Konfliktlinien werden sich dabei um den $>$ Grad der Zurichtung < natürlicher Strukturen und Materialien drehen (`Naturnähe< versus >Zurichtung $<$ ) und um die zugegebenermaßen grob vereinfachende Alternative zwischen einer Effizienzsteigerung, die entweder durch höhere Eingriffstiefe in Naturzusammenhänge erzielt wird, oder durch intelligentere Ausgeklügeltheit, durch ein kenntnisreiches, geschicktes, eingepaßt-mimetisches Bewegen zwischen den $\mathrm{Na}$ turkräften und Aufbauen auf natürlichen Strukturen ( $>$ Sophistication/Mitproduktivität $<$ ).

Im folgenden sollen als Beispiel für diese Konfliktlinien einige Ergebnisse des Gutachtens »Umwelteinflüsse Neuer Werkstoffe« vorge- stellt werden, das das IÖW im Auftrag des Büros für Technikfolgenabschätzung beim Deutschen Bundestag (TAB) durchgeführt hat.

\section{Überbetonung von Effizienzvorteilen}

Ein Teil dieses Gutachtens konzentriert sich auf die exemplarische Untersuchung eines Neuen Werkstoffs in einem spezifischen Anwendungsgebiet, auf Neue Keramiken in der Automobilbranche. Da über potentielle Umwelteinflüsse Neuer Werkstoffe bisher kaum publiziert wurde, führten wir persönliche Gespräche mit Fachleuten aus Forschung und Industrie. Wir erwarteten von dieser Befragung weniger vollständige Detailinformationen über qualitative und quantitative Umwelteinflüsse als vielmehr Eindrücke, mit deren Hilfe wir Umweltbelastungspotentiale von Neuen Keramiken und den Umgang der Befragten mit der Thematik zu erspüren hofften. Als Ergebnis dieser Befragung entwickelten wir Thesen, die in einer zweiten Befragungsrunde den Experten noch einmal vorgelegt wurden. Eine davon lautete: Kerami- 
(c) 20I0 Authors; licensee IÖW and oekom verlag. This is an article distributed under the terms of the Creative Commons Attribution Non-Commercial No Derivates License (http://creativecommons.org/licenses/by-nc-nd/3.o/), which permits unrestricted use, distribution, and reproduction in any medium, provided the original work is properly cited. 